\title{
Employ of multivariate analysis and chemometrics in cultural heritage and environment fields
}

\author{
Giovanni Visco $^{1} \cdot$ Pasquale Avino $^{1}$
}

Received: 10 April 2017 / Accepted: 3 May 2017 / Published online: 23 May 2017

(C) Springer-Verlag Berlin Heidelberg 2017

This Special Issue contains a selection of ten papers from the 5th edition of the biennial CMA4CH Meeting held in Rome, 14-17 December 2014, Italy. From the first edition, the Meeting is under the patronage of UNESCO, University of Rome "La Sapienza," the Italian Ministry of Cultural Heritage, Italian Chemical Society, the Italian Research National Council (CNR), and Federchimica (the association of chemical Italian stakeholders).

The guideline of the Meeting, which was also the main theme, was the "Employ of Multivariate Analysis and Chemometrics in Cultural Heritage and Environment Fields." CMA4CH meetings were born in 2006 for grouping and collecting researchers around the Mediterranean area.

In these 10 years and six editions, this serial meeting is really grown up welcoming people from Romania, Bulgaria, Germany, the USA, and, even, Iran, Brazil, and Cuba: it has produced a valuable amount of information and knowledge, contained in more than 400 oral and poster contributions in hot topics.

This edition presented some international researches representing 11 countries, and discussed in these 4 days are about the use of statistic, multivariate methods, and chemometric approaches for solving or, at least, for helping to solve environmental and cultural heritage problems. All long abstracts, collected in a proceeding book with ISBN, pass a peer review.

Responsible editor: Philippe Garrigues

Giovanni Visco

visco@uniromal.it

1 Department of Chemistry, Mat. Phy. Natural Science Faculty, Rome University, La Sapienza, Pl. Aldo Moro 5, 00185 Rome, Italy
The presentations, based on plenary lectures and oral and poster communications, covered different aspects, some of them are really innovative also from a technological point of view. The selection performed by the Scientific Committee was hard and we really apologize for all the researchers not present in this Special Issue: these Editors strongly believe that the other papers could also deserve publication. The high-quality level of Environmental Science and Pollution Research is the best assurance about the published papers.

With the background of the chemometric methods, the selected papers can be divided into two different sections: four papers are devoted to solving cultural heritage problems whereas six papers to environmental problems.

The articles regarding the cultural heritage issue are four and cover aspects related to the application of statistical methods to different case studies. Through the archaeometric investigations, Amadori et al. demonstrated that historical and stylistic approach should be helped by analytical data. The authors applied these considerations to Punic ceramics and potteries found in different Mediterranean sites for identifying the production site (Carthage). Following an international cooperation with Polish Academy of Sciences, Siani et al. stated that the indoor microclimate conditions in Mogila Abbey in Kraków are not suitable for the conservation of organic materials: the group used the analogue method as an operative tool to predict future environmental conditions. Sammartino's group reported a multivariate comparison of microclimate parameters in a historical site in Rome (Carcer Tullianum): the authors concluded that the data treatment is sufficient to highlight the differences between the presence and the absence of visitors in such important sites. In the other papers, the 
same group characterized the physic-chemical parameters of the plaster of a historical building in Cagliari (Sardinia) identifying the reasons of the degradation state.

In the second set of the articles presented in this Special Issue, the main topics addressed in the environmental field have regarded wood, water, soil, and air. The added value in some papers was the relevant effects of the findings on the human health, which is considered the final receptor of any chemical exposure of population. Ielpo et al. applied the principal component analysis (PCA), the cluster analysis (CA), the discriminant function analysis (DFA), and the radial basis function neural networks (RBF-NN) to a wide dataset of ground waters and soils collected in Apulian agricultural sites: the results point out the soil's fertility among Apulia provinces as well as that the groundwater pollution sources, mainly coming from agricultural practices and marine water intrusion, pressuring the sites are similar among the different provinces. D'Ambrosio et al. used the PCA for choosing among the 33 calculated hydrological indexes those which represent the main aspect of flow regime: by the selected parameters, they manage to derive generalization and information useful for river basin management. A paper which is at the border of the two issues in this meeting was presented by Pelosi et al., i.e., the study of the surface color and the relative chemical modifications in chestnut wood samples following wood aging. The authors extensively used the Fourier transform infrared spectrometry (FT-IR) and the hyperspectral imaging techniques and after statistical elaboration evidenced the strict correlation between lignin alteration and color change, an important process in the wooden material world and cultural heritage as well. Interesting contributions are proposed by researchers of the Italian institute devoted to health and safety at workplace, INAIL. Tranfo and colleagues assessed the levels of benzene and nicotine metabolites as exposure biomarkers on urine samples collected from a large population of non- and ex-smokers and smokers, evaluating simultaneously the oxidative damage to DNA/ RNA. In two consequent papers, Avino and Manigrasso depicted the dynamic of submicrometer particles, with a particular attention to the ultrafine particles, in urban areas and the relative aerosol dose deposited in the human respiratory tract: for the first time, this issue is deeply investigated. Starting from the concentration levels (almost $\left.7 \times 10^{10} \#\right)$ and investigating their relative dynamic, the authors stated that the particles deposited in the respiratory system are almost entirely made of ultrafine particles $(<100 \mathrm{~nm})$ and the right lung lobes are expected to be very susceptible to pneumo-pathologies.
Parallel to scientific significance, it is important to highlight the course on chemometric methods held in the same days. This class was of very high level: the past lectures came from Germany, Italy, the UK, and the USA, and in this edition, three famous chemometrician held lessons. In 3 days, all the chemometric methods were addressed and discussed with the implications in different fields. The lectures deal with application in material sciences, nanotechnology, physical, chemical sciences, and medical studies with high satisfaction by the students.

Finally, the guest editors would like to thank each author for her/his generous effort for contributing to the realization of this Special Issue and the journal's editors who handled the submissions and suggest all readers to be present in the 2018 edition of CMA4CH Meeting.

Rome 06 April 2017

Giovanni Visco

Pasquale Avino

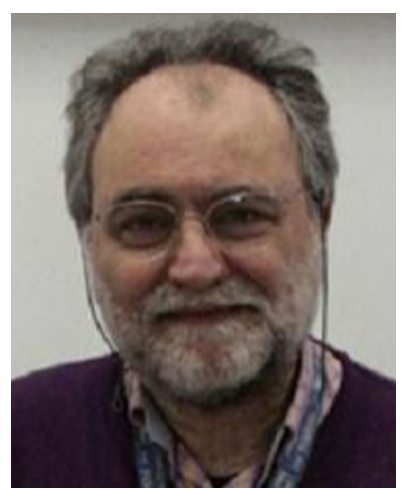

Giovanni Visco is currently an adjunct professor at the University $\mathrm{La}$ Sapienza in Rome, Italy. Since 2001, he teaches in the Degree Course "Technologies for the Preservation and Restoration of Cultural Heritage" of the Faculty of Mathematical, Physical and Natural Sciences, University of Rome La Sapienza. He is the creator, organizer, and coordinator of the biennial International Meeting $\mathrm{CMA} 4 \mathrm{CH}$ (Chemometrics and Multivariate Analysis applied to Cultural Heritage and Environment) from 2006 until today. He is the coordinator of teachers and lecturers in the Regional Training Course on Chemometrics-DataEvaluation of the International Atomic Energy Agency (IAEA), held in Tirana, Albania, 10-14 Dec. 2007, even with the lecturer activity. He is a guest editor of Special Issues, related to editions of the Meeting CMA4CH, in international journal such as Microchemical Journal, Chemistry Central Journal, Current Analytical Chemistry, and Environmental Science and Pollution Research. He is already teaching owner "Data processing and Chemometrics in the chemical and agro-food Automation Tools and Information in the product laboratory" at the University of Cassino; already an adjunct professor for the supplementary course "Applications of Computer in Laboratory" at La Sapienza University; already a contract professor in the supplementary course "Application of Computer in Analytical Chemistry Program" at La Sapienza University; already holds a research grant "Monitoring via screening and confirmatory methods, using sensors and biosensors, the quality of drinking water with the use of chemometric methods of calculation and multivariate", by La Sapienza University. He is a professional contract at the Department of Chemistry, La Sapienza University, "Data processing for the classification of archaeological findings using pattern recognition analysis." He is the author of about 60 publications for which the statistical treatment of the data was within its exclusive competence. 


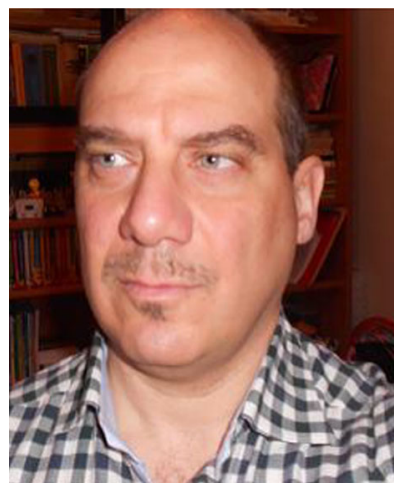

Pasquale Avino received his Master in Chemistry at the University of Rome "La Sapienza" in 1992 and his Ph.D. in Chemical Sciences at the University of Rome "La Sapienza" in 1997. From 1997 to 1998 , he was appointed as a post-graduate researcher in the Blake-Rowland's Laboratory at the University of California, Irvine (USA), studying the separation of non-methane compounds and the halocarbons and their behavior in atmosphere. In September 1997, he participated in the SONEX project funded by NASA on the determination of hydrocarbons in troposphere: for his fundamental support in such project, he was rewarded with the "Group Achievement NASA Award.” From 1999 until now, he has been appointed as researcher at the INAIL Research Center. Dr. Avino is involved in the Analytical Chemistry and Air Chemical Laboratory: his studies are devoted to both the development of analytical protocols for analyzing hazardous compounds in different environmental matrices and the different aspects of the atmospheric pollution but the main regard is the characterization of areas at high sensibility both residential (urban and suburban) and industrial sites. In 2003, Dr. Avino was the recipient of the Environmental Sapio Award for his research in the environmental field (particulate matter characterization, physical and chemical composition, health effects, etc.). From 2002 to 2005, he was ISPESL responsible in the European Project (FP VI) "Methodology to develop Acute Exposure Threshold Levels (ACUTEX) in case of chemical release": the project would increase methodologies and guidelines to set up acute exposure levels in case of accidental release of toxic chemical substances. From 2004, Dr. Avino is an Adjunct Professor of Analytical Chemistry and Environmental Chemistry at the University of Molise, Campobasso. Further, Dr. Avino attends to many National and International Committee Panels for discussing environmental issues and is the author of over 100 papers published in international journals and books. 